\title{
Dietas insulina-estimulantes para las hembras porcinas
}

\author{
Silva, M.C. ${ }^{1}$; Muñoz Luna, A. ${ }^{2}$; Kiefer, C. ${ }^{\text {; }}$ Ramis Vidal, G. ${ }^{2}$; Alencar, S.A.S. ${ }^{1}$ y Cambra-Bort, J.M. ${ }^{2}$
}

'Programa de Postgrado en Ciencia Animal. Universidad Federal de Mato Grosso do Sul. Brasil. 2Departamento de Producción animal. Facultad de Veterinaria. Universidad de Murcia. España.

\section{PALABRAS CLAVE ADICIONALES}

Insulina.

Cerdas.

Almidón.

\section{RESUMEN}

Esta revisión bibliográfica tiene como objetivo presentar los efectos de la insulina modulada por la dieta en el rendimiento reproductivo y productivo de las cerdas. La insulina es una hormona que tiene papel de intermediario entre la nutrición y la reproducción, causando la síntesis de gonadotropinas, $\mathrm{FSH}$ y $\mathrm{LH}$, a través de efectos directos sobre los ovarios. Los niveles de insulina endógena pueden estar influidos tanto por los nutrientes ingeridos como por la fuente de energía. Los estudios científicos han demostrado que las dietas donde los lípidos son la fuente principal de energía no producen mejoras en la condición corporal de las cerdas reproductoras durante la lactancia y tampoco en el desarrollo reproductivo después del destete. De hecho, puede empeorar la condición catabólica, debido al aumento de grasa en la producción de leche. Por otra parte, aumentar el contenido de carbohidratos durante la lactación puede mejorar el balance de energía, la secreción de gonadotropina y los perfiles de insulina y glucosa. Se ha demostrado que es posible modular los niveles de insulina en plasma mediante el consumo de hidratos de carbono complejos, tales como el almidón, que puede producir picos más prolongados de glucosa en comparación con los carbohidratos simples como dextrosas o sacarosas, promoviendo así el desarrollo folicular de los ovocitos, a partir de la síntesis de la hormona luteinizante a nivel en el ovario.

\section{Insulin-stimulating diets for sows}

\section{INFORMATION}

Cronología del artículo.

Recibido/Received: 05.07.2016

Aceptado/Accepted: 17.04.2017

On-line: 15.10 .2017

Correspondencia a los autores/Contact e-mail:

camillamsazoo@gmail.com

\begin{abstract}
SUMMARY
This bibliographic review aims to introduce the effects of insulin modulated by diet in the productive and reproductive efficiency of sows. Insulin is an hormone that plays an intermediary role between nutrition and reproduction, causing the synthesis of gonadotropins, FSH and $\mathrm{LH}$, through the direct effects of ovaries. Endogenous insulin levels may be influenced by nutrients and energy source. Scientific studies have shown that diets, in which lipids are the main source of energy, do not show improvements in the body condition of sows during lactation and reproductive development after weaning. In fact, catabolic condition can get worse due to the increase of fat in milk production. Moreover, increasing the carbohydrate content during lactation can improve energy balance, gonadotropin secretion and insulin and glucose profiles. Research results show that it is possible to modulate the levels of plasma insulin by consuming complex carbohydrates such as starch. This nutrient can produce longer glucose peaks compared to simple carbohydrates such as dextrose or saccharose carbon, promoting follicular oocyte development, from the synthesis of luteinizing hormone at an ovaric level.
\end{abstract}

\section{INTRODUCCIÓN}

Las interacciones entre la nutrición y la reproducción se han estudiado durante muchos años en diversas especies animales con evidencias de que la función reproductiva puede verse afectada por la ingesta inadecuada de nutrientes y energía. De acuerdo con Pupa y Barroca (2013), la nutrición durante la gestación influye en el desarrollo de la hembra, el peso, el tamaño y la uniformidad de la camada, además de influir en la productividad durante el período de lactancia y el intervalo destete-estro. 
La alimentación de las cerdas durante la gestación debe afrontar las necesidades de mantenimiento corporal, crecimiento del tejido uterino, de la mama y crecimiento fetal. El nivel de energía es el factor más crítico en esta etapa, y determina los resultados productivos de la cerda. Se ha demostrado que la fuente de energía alimentaria ejerce influencias en las respuestas insulínicas y la liberación de LH y progesterona, señal de que las dietas donde la fuente de energía es un hidrato de carbono, son potencialmente más beneficiosas en comparación con las dietas cuya fuente de energía son grasas o aceites, ya sean de origen animal o vegetal, además de otras fuentes de energía como almidón, en relación con el rendimiento reproductivo de las cerdas (Ferguson et al., 2003; Brustolini et al., 2004; Fontes et al., 2014). La inclusión de un $20 \%$ de almidón de maíz como fuente de energía en las cerdas jóvenes, mejora la eficiencia reproductiva de los animales (Machado et al., 2008).

El almidón en la dieta de las hembras puede aumentar los niveles plasmáticos de insulina, lo que produce la liberación de la hormona luteinizante, afectando la foliculogénesis (Quesnel, 2009). La acción de la insulina sobre la reproducción aún no está clara, por lo que es posible que las estrategias nutricionales repercutan en la reproducción, no sólo a través de mediadores aislados, como la insulina, sino a través de todo el status metabólico del animal (Penz Junior et al., 2009).

Por lo tanto, el establecimiento correcto de estrategias nutricionales para cerdas reproductoras se vuelve importante, ya que permite el equilibrio endocrino y fisiológico, refleja una mayor supervivencia de los embriones, produce un desarrollo satisfactorio de las glándulas mamarias y fetos, una recuperación adecuada de las reservas corporales de las multíparas, un crecimiento corporal correcto en cerdas jóvenes y un mayor número de lechones al nacer (Almeida et al., 2001; Zangeronimo et al., 2013; Corassa et al., 2014). En este contexto, esta revisión engloba aspectos que relacionan la insulina modulada por la dieta y sus efectos sobre las características reproductivas y productivas de la reproductora porcina.

\section{FUNCIONES Y CONTROL DE LA INSULINA}

La insulina desempeña un papel central en la regulación de la homeostasis de la glucosa, y actúa de manera coordinada en eventos celulares que regulan el metabolismo y el desarrollo del crecimiento en una amplia variedad de tipos celulares, incluyendo el ovario (Haber et al., 2001). Es secretada por las células beta en los islotes de Langerhans del páncreas (Waeber et al., 1997), en respuesta a niveles de glucosa en plasma, y en menor medida aminoácidos (arginina y leucina), y algunos ácidos grasos (ácido oléico y palmítico). Es controlada por hormonas pancreáticas como el glucagón y la somatostatina y neuropéptidos gastrointestinales liberados durante la digestión (Penz Junior et al., 2009).

\section{ACCIÓN DE LA INSULINA EN LA REPRODUCCIÓN}

En el ovario, la insulina es un modulador importante del desarrollo folicular, la esteroidogénesis, la maduración de ovocitos y el subsiguiente desarrollo embrionario (Yaseen et al., 2001). La insulina también conduce a un aumento en el número de folículos primarios y una tasa más baja en la atresia folicular y por lo tanto un aumento en la tasa de ovulación (Almeida et al., 2001; Chaves et al., 2011). El efecto de la insulina en el ovario de los mamíferos se puede demostrar por la amplia presencia de receptores en el tejido del ovario, incluyendo las células de la granulosa, la teca, el estroma y el ovocito (Myers et al., 1991).

De acuerdo con Penz Junior et al. (2009), la acción de la insulina en la reproducción todavía no esta clara, mientras que algunos estudios evidencian la ausencia de su efecto en la reproducción o incluso un efecto negativo; Cox (1997) describe que la variación en los resultados obtenidos acerca de la insulina sobre la reproducción porcina, pueden estar relacionados con la producción de una señal metabólica negativa debido al uso prolongado de la insulina inyectable.

Incluso los autores señalan que con sólo el aumento de la ingesta de alimentos desde el destete al estro podemos conseguir aumentar los niveles de insulina, aunque las circunstancias metabólicas de los animales pueden interfer en las respuestas evaluadas. Así pues, las hembras alimentadas con dietas con alto porcentaje de grasa durante todo el período de gestación puede presentar una intensa situación anabólica, lo que resulta en resistencia a la insulina en los tejidos, reduciendo el número o la afinidad de los receptores de insulina en el alvos periféricos, por ejemplo, en los adipocitos (Weldon et al., 1994; Azain, 2000). Al final, el resultado de la diabetes gestacional, es la variabilidad intrínseca para respuestas reproductivas en general (Machado et al., 2008).

La insulina actúa en la reproducción para regular la síntesis de neurotransmisores de GnRH y, en consecuencia, sobre el control de la secreción de gonadotropina, y en especial de la liberación de LH por la hipófisis, junto con los receptores de IGF-I e IGF-II que están presentes en las células foliculares, concretamente en las capas de la granulosa y de la teca) (Kawauchi y Sower, 2006). La insulina y el IGF-I disminuyen el grado de atresia durante la fase folicular (Matamoros et al., 1990). La unión de la insulina a sus receptores genera una serie de procesos metabólicos, el más importante es la estimulación del transporte de glucosa al interior de las células, siendo esta, la principal fuente de energía para el ovario (Souza et al., 2009).

Esta hormona también afecta a la tasa de ovulación y a la producción de IGF-I por los folículos (Cox, 1997), existiendo una correlación positiva entre las concentraciones de insulina y la frecuencia de los pulsos de LH durante la lactancia (Tokack et al., 1992; Quesnel et al., 1998).

Según Booth et al. (1994), una alimentación excesiva aumenta la actividad de la aromatasa y el desarrollo folicular, debido al aumento de los niveles de insulina. Por lo tanto, el efecto positivo del manejo del flushing en las cerdas jóvenes sobre la tasa de ovulación, está mediado por los niveles de insulina en plasma, que afectarán al reclutamiento, la selección y el crecimiento de los folículos (Bortolozzo et al., 2007). 
En las cerdas, el nivel en sangre de IGF-I es expresado en las células de la granulosa del folículo con 2-8 $\mathrm{mm}$ diámetro. Sin embargo, el nivel de IGF-II es mayor en las células de la teca de los folículos con $6 \mathrm{~mm}$, y ésta permanece alta hasta que se produce el pico de LH, lo que indica una cierta función en la ovulación y/o lutenización (Webb et al., 2007). El IGF-I y la insulina también interactúan con la FSH para estimular las células de la granulosa para producir estradiol.

Según Mao et al. (2004), para los cerdos, el IGF-I desempeña un papel importante en la foliculogénesis, actuando en la supresión de la apoptosis y promoviendo la replicación de las células de la granulosa de los folículos preantrales, con aplicación potencial en el desarrollo de los folículos in vitro, cuyos oocitos son adecuados para la fertilización y el desarrollo embrionario. Demeestere et al. (2004), observaron que, utilizando $50 \mathrm{mg} / \mathrm{ml}$ de IGF-I en cerdas, se produjo un crecimiento folicular que estimuló la proliferación de células de la granulosa y previno la apoptosis de los folículos preantrales cultivados durante cuatro días en presencia de suero.

\section{EFECTOS DE LA NUTRICIÓN EN LA REPRODUCCIÓN}

La comprensión de la interacción entre la nutrición y la reproducción es esencial para el desarrollo de un programa nutricional adecuado, que refleje el desarrollo adecuado de las cerdas reproductoras, y que permita la plena utilización del potencial genético de estos animales, conduciendo a un aumento de peso de los lechones al nacer y a una redución la mortalidad de los recién nacidos (Zangeronimo et al., 2013).

La nutrición afecta a la función reproductiva a través de la síntesis de algunas hormonas y metabolitos, cuya liberación esta condicionada principalmente por los niveles de energía y nutrientes ingeridos por los cerdos. Los cambios en los niveles de nutrientes son acompañados por cambios en las concentraciones circulantes de algunas hormonas como la insulina, la hormona del crecimiento, IGF-I, la leptina, el cortisol, la hormona tiroidea y ciertos nutrientes tales como glucosa, ácidos grasos libres y aminoácidos (Penz Junior et al., 2009). Mientras que a nivel sistémico, el estado metabólico del animal afecta a la modulación neuronal hipotalámica, estimulando la secreción de gonadotropina pituitaria a través de varias hormonas y neuropéptidos, que actuaran a nivel del ovario regulando el desarrollo folicular y la esteroidogénesis por medio de la hormona de crecimiento folicular, IGF-I, y mediadores locales del ovario (Cortez y Toniolli, 2012).

Dentro de estos factores, el requerimiento de energía tiene una mayor importancia en la relación nutrición y reproducción (Van Den Brand et al., 2000; Silva et al., 2014). Por lo tanto, las fuentes de energía en las dietas de hembras reproductoras, influyen en la secreción de insulina y IGF-I (Carrión y Medel, 2001), que tiene un efecto directo sobre las gonadotropinas FSH y LH y sobre los ovarios (Machado et al., 2008). Los niveles altos de almidón en la dieta de las cerdas, producen un aumento en los niveles de insulina en plasma, que pueden afectar a los niveles de glucosa, generando una mayor sensación de bienestar y la saciedad en las hembras (Cox, 1997; Wientjes et al., 2013).
El manejo del flushing en cerdas jovenes, utilizando almidón de maíz como fuente de energía, aumenta la tasa de ovulación, el peso total de los ovarios, el número total de embriones viables, el tamaño medio de los embriones, el área media de la placenta y el peso medio de las placentas (Fontes et al., 2014). El peso de la placenta se correlaciona positivamente con embrión/ peso del feto, es decir, placentas más pesadas pueden mantener embriones/fetos más pesados (Town et al., 2004). A su vez, los lechones más pesados al nacer, acaban teniendo un mayor peso al destete (Alvarenga et al., 2013). Al evaluar el efecto de diferentes fuentes de energía en la dieta de cerdas jóvenes, Machado et al. (2008), se observó que en las dietas de flushing basadas en almidón de maíz, había un aumento en la tasa de ovulación. En un estudio similar Almeida et al. (2014), encontraron que las cerdas alimentadas con almidón de maíz, tuvieron mayores tasa de ovulación, número de embriones, peso del embrión y peso de la placenta, en comparación con aquellas dietas de flushing basadas en aceite de soja como fuente de energía. Este resultado sugiere que los efectos beneficiosos de la insulina no se aprecian sólo en el reclutamiento folicular, sino también en la calidad de los folículos. Por lo tanto, los folículos más desarrollados pueden generar ovocitos de mejor calidad y, a su vez, embriones viables (Geisert et al., 2002).

Por su parte, Kemp et al. (1995), no encontraron un efecto entre la fuente de energía y la supervivencia embrionaria, pero encontraron un aumento en los niveles de progesterona en el proestro, y un aumento en la secreción pulsátil de LH en las cerdas alimentadas con dietas que contiene $20 \%$ de almidón en comparación con el grupo tratado con una dieta basada en lípidos ( $0,2 \%$ de aceite de soja).

En un estudio con la inclusión de un $20 \%$ de almidón de maíz y un $8 \%$ de aceite de soja como fuente de energía para las cerdas, Almeida et al. (2014), verificaron picos de insulina en los días 14 y 21 del ciclo estral (figura 1a y 1b), y se observó que las dietas enriquecidas con almidón de maíz presentaban los picos más altos. Los investigadores también encontraron que ambas fuentes de energía no mostraron diferencias en cuanto a la duración de la concentración de insulina; sin embargo, cerdas jóvenes alimentadas con almidón de maíz tuvieron concentraciones numéricamente mayores (figura 1a y 1b) (Almeida et al., 2014).

En un estudio para evaluar los diferentes niveles de inclusión del almidón de maíz asociados con dextrosa plus en polvo en la dieta de las cerdas, durante el intervalo destete-estro, Wientjes et al. (2013), verificaron, que la dextrosa junto con el almidón en la dieta fueron eficaces para la estimulación de la secreción de insulina postprandial, con picos de duración a largo plazo en la concentración plasmática, pero no se observaron efectos sobre la estimulación de IGF-I durante los tres días después del destete.

Al evaluar el efecto de diferentes fuentes de energía de origen lipídico o de hidratos de carbono, para las cerdas durante la gestación y la lactancia, se observó que las diferentes dietas no afectaron el intervalo destete-estro ni al peso de los lechones al nacer (Quiniou 

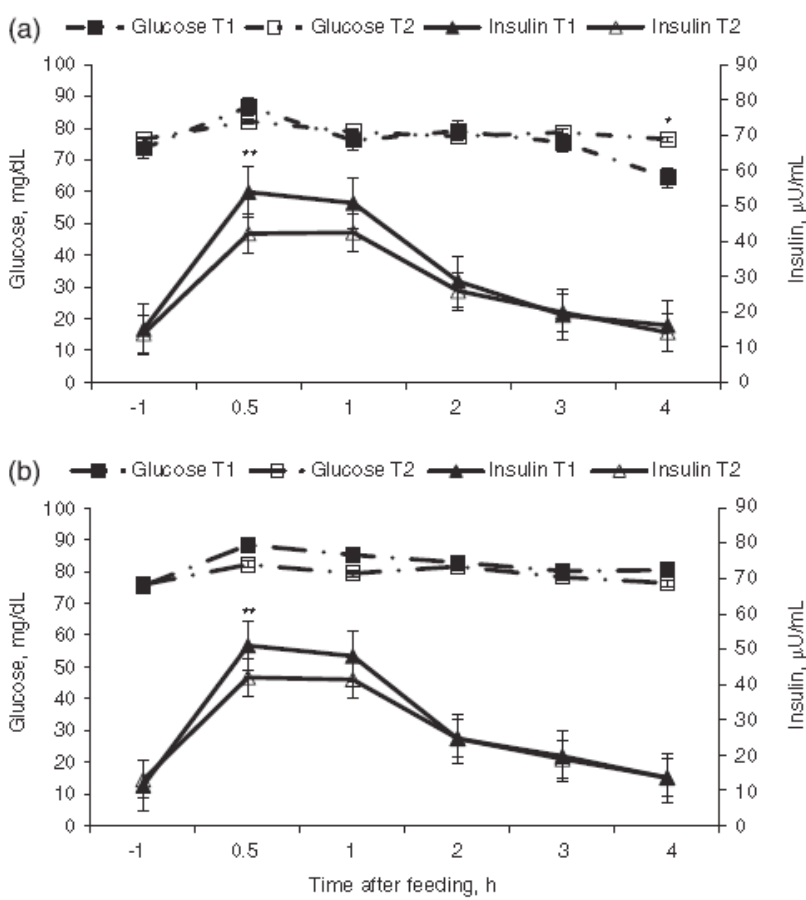

Figura 1. Concentraciones de glucosa en la sangre y la insulina en plasma pre y post-prandial en 14 (a) y 21 (b) días del ciclo estral en las cerdas jóvenes, alimentadas con dietas enriquecidas con almidón de maíz (T1) o aceite vegetal (T2) durante la fase lútea tardía del ciclo. Cerdas jóvenes T1 tiene una menor concentración de glucosa en sangre después de $4 \mathrm{~h}$ de la alimentación en el día 14 del ciclo $(\mathrm{p}<0,05 ; \mathrm{a})$; Cerdas jóvenes T1 mostraron niveles elevados de insulina 30 minutos después de la alimentación en los días 14 y $21(\mathrm{p}<0,05, \mathrm{~b})$. Adaptado de Almeida et al. (2014) (Pre- and postprandial blood glucose and plasma insulin concentrations on days 14 (a) and 21 (b) of the estrous cycle in gilts, fed either corn starch (T1) or vegetable oil-rich (T2) diets during the late luteal phase of the cycle. ${ }^{*}$ T1 gilts presented a lower blood glucose concentration $4 \mathrm{~h}$ after feeding on day 14 of the cycle $(p<0,05$, y b). Adapted from Almeida et al. (2014)).

et al., 2005). Sin embargo, la tasa de crecimiento de los lechones en la fase de acabado se vió afectada por el tipo de alimento utilizado como fuente energética en la alimentación; así pues, los lechones procedentes de los lotes en los que las hembras fueron alimentados con dietas con un 5\% de aceite de soja, presentaron mayor ganancia de peso en el período, en comparación con el lote proveniente de hembras alimentadas con dietas que contenían un 11\% de almidón de maíz (3,22 vs 3,02 $\mathrm{kg} /$ dia, $\mathrm{p}<0,05)$.

Van Den Brand et al. (2000), evaluaron el efecto de las fuentes de energía para las cerdas primíparas lactantes, y pudieron constatar que hembras alimentadas con almidón de maíz presentaban una concentración de glucosa e insulina en plasma más altas que en comparación con hembras alimentadas con grasa de origen bovino. Los autores indicaban que las hembras cíclicas alimentadas con almidón de maíz pueden presentar una alta concentración de insulina hasta 4,5 horas después de la alimentación. Quesnel et al. (2008), encontraron que las cerdas con picos altos y prolongados de la insulina después de una prueba de tolerancia a la glucosa, tuvieron folículos más desarrollados, más grandes y más numerosos.
Tabla I. Contenido de almidón de algunos alimentos utilizados en dietas para cerdos (Starch content of some foods used in diets for pigs).

\begin{tabular}{lcc}
\hline Alimentos & \% Almidón & \% Almidón ${ }^{2}$ \\
\hline Arroz & 74,45 & 75,19 \\
Cebada & ---- & 50,21 \\
Centeno & ---- & 59,34 \\
Guisante & ----- & 43,46 \\
Harina de avena & ---- & 39,06 \\
Harina de soja & 3,00 & 1,890 \\
Maíz & 62,66 & 67,44 \\
Sorgo & 63,24 & 70,05 \\
Trigo & 31,35 & 21,83 \\
Triticale & 55,25 & 64,31 \\
\hline
\end{tabular}

${ }^{1}$ Rostagno et al. (2011); ${ }^{2} \mathrm{NRC}(2012)$

El diámetro de los folículos dos días después del destete tenderá a ser mayor en las cerdas alimentadas con almidón de maíz, comparado con las alimentadas con grasa, concretamente 3,8 frente a 3,1 mm $(p<0,01)$, respectivamente (Van Den Brand et al., 2000). Según Silva et al. (2014), los folículos más grandes tienes más receptores de LH, reduciendo la posibilidad que se produzca atresia, por lo tanto, la población de los folículos se vuelve más uniforme, lo que resulta en una mayor calidad de los ovocitos y en embriones más uniformes.

\section{AlimENTOS INSULINA ESTIMULANTES}

Los estudios con cerdas muestran que los niveles de insulina en plasma pueden estar influenciados por la composición de la dieta (Martínez et al., 2013). El aumento de los niveles plasmáticos de insulina en hembras primíparas lactantes alimentadas con dietas enriquecidas con aceites y azúcares fueron verificados por Chen et al. (2013), observando además un mayor aumento, con el enriquecimiento en azúcares en la ración, frente a la incorporación de aceite.

En el metabolismo, la insulina estimula la distribución intracelular de la glucosa a través de la activación de la glucógeno sintasa y de la inhibición de la glucogenolisis, simultáneamente con la activación de la glucólisis y la lipogénesis y la utilización de la glucosa como combustible oxidativo (Fonseca- Alaniz et al., 2006). Por lo tanto, teniendo en cuenta la importancia de la insulina y del IGF-I en las interacciones entre la nutrición y la reproducción de las hembras, se puede inferir que las dietas enriquecidas con alimentos que promueven el aumento de los niveles plasmáticos de insulina y el IGF-I pueden mejorar la fertilidad, reducir el intervalo del destete al estro, y en última instancia, promover una mejor uniformidad y peso de los lechones al nacer.

El almidón produce un aumento en los niveles de insulina en plasma, pues se clasifica como un carbohidrato soluble, y es fácilmente digestible en el intestino delgado (Gobesso et al., 2009; Yin et al., 2012). En ciertas especies de plantas es un carbohidrato de reserva y es abundante en granos de cereales (40 a 90\% del peso seco), legumbres (30 a 50\% del peso seco), tubérculos 
Tabla II. Comportamiento productivo de las cerdas alimentadas con diferentes fuentes y niveles de almidón (Productive performance of sows fed on different sources and levels of starch).

\begin{tabular}{|c|c|c|c|c|c|c|c|c|c|c|}
\hline \%Almidón & ${ }^{*} \mathrm{CDR}$ kg & $\begin{array}{c}\text { CD } \\
\text { almidón } \\
\text { kg }\end{array}$ & $\begin{array}{c}\text { CD } \\
\text { energía } \\
\text { Mcal/kg }\end{array}$ & $\begin{array}{l}\text { *Pérdida } \\
\text { peso Lac. }\end{array}$ & $\begin{array}{l}{ }^{*} \text { Perda } \\
\text { EGD mm }\end{array}$ & IDC & $\begin{array}{c}\text { Lechones } \\
\text { vivos }\end{array}$ & $\begin{array}{l}\text { Peso } \\
\text { parto }\end{array}$ & $\begin{array}{c}\text { Peso } \\
\text { destete }\end{array}$ & Artículo \\
\hline 32,3 & 6,61 & 2,14 & 19,74 & 23,0 & 3,6 & 4,0 & 13,5 & 1,50 & 9,06 & 1 \\
\hline 30,1 & 5,50 & 1,65 & 16,54 & 17,5 & 3,8 & - & 12,3 & 1,42 & 9,18 & 2 \\
\hline 28,0 & 5,16 & 1,45 & 15,97 & 21,0 & 3,8 & 6,0 & 13,1 & 1,25 & 10,25 & 3 \\
\hline 19,0 & 6,18 & 1,30 & 18,63 & 9,8 & 1,7 & 6,0 & 10,4 & 1,38 & 6,38 & 4 \\
\hline 16,5 & 6,06 & 1,10 & 18,07 & 17,4 & 3,4 & 4,6 & 13,0 & 1,40 & 8,13 & 5 \\
\hline 13,7 & 6,09 & 0,80 & 18,52 & 14,3 & 2,6 & 5,6 & 11,2 & 1,39 & 7,09 & 4 \\
\hline
\end{tabular}

1.Quiniou et al. (2006); 2. Boone et al. (2001); 3. Thingnes et al. (2013); 4. Guillemet et al. (2007); 5.Zhao et al. (2014); Composición nutricional de las dietas de acuerdo a las recomendaciones nutricionales de NRC(2012). CDR: consumo diario de ración; pérdida peso lac.: pérdida de peso en la lactancia; pérdida EGD mm: pérdida en el espesor de grasa dorsal.

(65 a 85\% del peso seco) y frutos inmaduros o verdes (40 a 70\% del peso seco) (Lajolo y Menezes, 2006; Monte y Nascimento, 2013). Entre los ingredientes utilizados en la alimentación porcina (tabla I), la cebada, el trigo, el maíz, el sorgo, la mandioca, el triticale y el centeno son los que tienen un alto contenido de almidón, por lo tanto puede enriquecer las dietas de hembras reproductoras.

Por otro lado, hay poca información en la literatura científica que relacione el nivel apropiado de inclusión de almidón en la dieta con los parámetros reproductivos y productivos de cerdas. Dado que muchos alimentos con alto contenido de almidón se usan en las formulaciones, es relevante el conocimiento de los niveles apropiados de inclusión de esta fuente de energía en las dietas de las cerdas (tabla II).

El exceso en el consumo de energía puede dar lugar a una serie de daños, tales como alta mortalidad embrionaria, reducción en el desarrollo mamario, cerdas grasas con problemas del aparato locomotor, reducción del consumo durante la lactancia y disminución del rendimiento de la lactancia (Kim et al., 2001). Por otro lado, el consumo insuficiente o desequilibrado provoca una disminución de las reservas de grasa corporal al parto o al deteste y, generalmente, retrasa la presentación del estro y baja la tasa de concepción (Pupa y Barroca, 2013). Por lo tanto, para permitir un aumento de la productividad de las hembras, debe considerarse el consumo diario de la ración, en relación al consumo de nutrientes, cuya relación debe atender a las exigencias de mantenimiento y de producción. Por otra parte, las actuales líneas genéticas tienen una baja capacidad física de consumo diario de alimento, por lo que es necesario incluir fuentes con una mayor densidad de energía como aceites y grasas para tratar de cumplir los requisitos nutricionales de las hembras (Sauber et al., 1994).

\section{CONCLUSIONES}

La estimulación de la secreción de insulina a través de la nutrición ha demostrado ser una alternativa eficaz para que las cerdas puedan expresar su potencial genético, y mejorar la fertilidad, reduciendo el intervalo destete-estro y promoviendo una mayor uniformi- dad y peso de los lechones al nacer. Tras esta revisión no es posible hacer una recomendación en relación con el nivel mínimo de inclusión de almidón en las dietas de las hembras. Deben llevarse a cabo nuevos estudios para evaluar no sólo el nivel de energía en la dieta de las cerdas, sino también las interacciones entre los niveles de energía, los niveles de inclusión y fuentes de almidón utilizados y los niveles de lípidos sobre el rendimiento reproductivo.

\section{BIBLIOGRAFÍA}

Almeida, F.R.; Mao, J.; Novak, S.; Cosgrove, J.R. y Foxcroft, G.R. 2001. Effects of different patterns of feed restriction and insulin treatment during the luteal phase on reproductive, metabolic, and endocrine parameters in cyclic gilts. J Anim Sci, 79: 200-212.

Almeida, F.R.C. L.; Machado, G.S.; Borges, A.L.C.C.; Rosa, B.O.y Fontes, D.O. 2014. Consequences of different dietary energy sources during follicular development on subsequent fertility of cyclic gilts. Animal, 8: 293-299.

Alvarenga, A.L.N.; Chiarini-Garcia, H.; Cardeal P.C.; Moreira L.P.; Foxcroft, G.R.; Fontes, D.O. y Almeida, F.R. 2013. Intra-uterine growth retardation affects birth weight and postnatal development in pigs, impairing muscle accretion, duodenal mucosa morphology and carcass traits. Reprod Fert Develop, 25: 387-395.

Azain, M.J. 2000. Fat in swine nutrition. In: Lewis, J.A and Shothern, L.L. (Eds). Swine nutrition. $2^{a}$ ed. CRC Press. Boca Raton. FL. 1032 pp.

Boone, C.; Cadoret, A.; Pére, M.C.; Étienne, M. y Mourot, J. 2001 . Effets du taux et de la nature des lipides du régime de gestation et de lactation des truies sur le développement des tissus adipeux des porcelets. J Rech. Porcine, 33: 157-163.

Booth, P.J.; Craigon, J. y Foxcroft, G.R. 1994. Nutritional manipulation of growth and metabolic and reproductive status in prepubertal gilts. J Anim Sci, 72: 2415-2424.

Bortolozzo, F.P.; Mellagi, A.P.G.; Amaral Filha, W.S. y Wentz, I. 2007. Fatores que influenciam no tamanho da leitegada. In: Bortolozzo, F.P. and Wentz, I. A fêmea suína gestante. $1^{a}$ ed. Grafica da UFRGS. Porto Alegre. RS. 150 pp.

Brustolini, P.C. Silva, F.C.O.; Donzele, J.L.; Veloso, J.A.F.; Fontes, D.O. y Kill, J.L. 2004. Efeitos de diferentes fontes lipídicas e níveis de energia sobre o desempenho reprodutivo de marrãs. Arqs Bras Med Vet Zoot, 56: online version.

Chaves, R.N. Saraiva, M.V.A.; Alves, A.M.C.V. y Figueiredo, J.R. 2011. Implicações da insulina na função ovariana e desenvolvimento embrionário. Acta Vet Bras, 5: 136-146.

Corassa, A. Prezotto, R.T.; Komiyama, C.M.; Moreira, P.S.A. y Araújo, C.V. 2014. Farelo de biscoito na alimentação de porcas em lactação. Pesqui Agropecu Trop, 44: 42-49. 
Chen, T. Y.; Stott, P.; O'leary, S.; Athorn, R.Z.; Bouwman, E.G. y Langendiik, P. 2013. Effects of pre-weaning dietary substitutions on plasma insulin and glucose profiles in primiparous sows. J Anim Physiol Anim Nutr, 97: 807-812.

Cortez, A.A. y Toniolli, R. 2012. Aspectos fisiológicos e hormonais da foliculogênese e ovulação em suínos. Rev Bras Reprod Anim, 36: 163-173.

Cox, N.M. 1997. Control of follicular development and ovulation rate in pigs. J Reprod Fertil, 52: 31-46.

Demeestere, I.; Gervy, C.; Centner, J.; Devreker, F.; Englert, Y. y Delbaere, A. 2004. Effect of insulin-like growth factor-I during preantral follicular culture on steroidogenesis, in vitro oocyte maturation, and embryo development in mice. Biol Reprod, 70: 1664-1669.

Carríon, D. y Medel, P. 2001 . Interacción nutrición reproducción en ganado porcino. In: XVI Curso de Especialización FEDNA. http://www.ucv. ve/fileadmin/user_upload/facultad_agronomia/Nutrici\%C3\%B3nReproducci\%C3\%B3n_en_Porcinos.pdf (05/09/2015).

Ferguson, E.M.; Ashworth, C.J.; Edwards, S.A.; Hawkins, N.; Hepburn, N. y Hunter, M.G. 2003. Effect of different nutritional regimens before ovulation on plasma concentrations of metabolic and reproductive hormones and oocyte maturation in gilts. Reprod, 126: 61-71.

Fontes, D.O.; Machado, G. y Fernandes, I.S. 2014. Flushing nutricional em leitoas: Fundamentos técnicos e aplicação prática. In: Associação de criadores de suínos - ABCS (Eds). Produção de Suínos: Teoria e Prática. $2^{a}$ ed. Coordenação Técnica Integrall Soluçães em Produção Animal. Brasília. DF. 908 pp.

Fonseca-Alaniz, M.H.; Takada, J.; Alonso-Vale, M.I.C. y Lima, F.B. 2006. $\mathrm{O}$ tecido adiposo como centro regulador do metabolismo. Arq Bras Endocrinol Metab, 50: 216-229.

Geisert, R.D. y Schmitt, R.A.M. 2002. Early embryonic survival in the pig: Can it be improved? J Anim Sci, 80: E54-E65.

Guillemet, R.; Hamard, A.; Quesnel, H.; Pére, M.C.; Etienne, M.; Dourmad, J.Y. y Meunier-Salau" n, M.C. 2007. Dietary fibre for gestating sows: effects on parturition progress, behaviour, litter and sow performance. animal, 1: 872-880.

Gobesso, A.A.O.; Etchichury, M. y Tosi, H. 2009. Resposta plasmática de glicose e insulina em eqüinos alimentados com diferentes fontes de amido. Braz J Vet Res Anim Sci, 46: 324-331.

Haber, E.P.; Curi, R.; Carvalho, C.R.O. y Carpinelli, A.R. 2001. Secreção da Insulina: Efeito Autócrino da Insulina e Modulação por Ácidos Graxos. Arq Bras Endocrinol Metab, 45: online version.

Kawauchi H. y Sower S.A. 2006. The dawn and evolution of hormones in the adenohypophysis. Gen Comp Endocrinol, 148: 3-14.

Kemp, B.; Soede, N.M.; Helmond, F.A. y Bosch, M.W. 1995. Effects of ennergy source in the diet on reproductive hormones and insulin during lactation and subsequent estrus in multiparous sows. J Anim Sci, 73: 3022-3029.

Kim, S. W.; Easter, R.A. y Hurley, W.L. 2001. The regression of unsuckled mammary glands during lactation in sows: The influence of lactation stage, dietary nutrients, and litter size. J Anim Sci, 79: 2659-2668.

Lajolo, F.M. y Menezes, E.W. 2006. Carbohidratos en alimentos regionales iberoamericanos. EDUSP. São Paulo. SP. 646 pp.

Mao, J.; Smith, M.F.; Rucker, E.B.; Wu, G.M.; Mccauley, T.C.; Cantley, T.C.; Prather, R.S.; Didion, B.A. y Day, B.N. 2004. Effect of epidermal growth factor and insulin-like growth factor I on porcine preantral follicular growth, antrum formation, and stimulation of granulosal cell proliferation and suppression of apoptosis in vitro. J Anim Sci, 82: 1967- 1975.

Machado, G.S.; Fontes, D.O.; Almeida, F.R.C.L.; Borges, A.L.C.C.; Silva, M.A.; Oliveira, V.F. y Silva, T.C.T. 2008. Efeitos de diferentes fontes de energia sobre taxa ovulatória, fertilidade e sobrevivência embrionária em marrãs cíclicas. Arqu Bras Med Vet Zoo, 60: 600-606

Martínez, S.; Campos, C.; Madrid, J.; Cerón, J.J.; Orengo, J.; Tvarijonaviciute, A.; Valera, L. y Hernández F. 2013. Conocimiento actual de las hormonas reguladoras de la ingestión de alimentos en la especie porcina. An Vet Murcia, 29: 7-22.
Matamoros, I.A.; Cox, N.M. y Moore, A.B. 1990. Exogenous insulin and additional energy affect follicular distribution, follicular steroid concentrations, and granulosa cell human chorionic gonadotropin binding is swine. Biol Reprod, 43: 1-7.

Monte, F.V. y Nascimento, K.O. 2013. Associação do consumo do amido resistente na prevenção e tratamento do diabetes mellitus tipo 2. Rev Verde, 8: 12-19.

Myers, M.G.; Backer, J.M.; Siddle, K. y White, M.F. 1991. The insulin receptor functions normally in Chinese hamster ovary cells after truncation of the $C$ terminus. J Biol Chem, 266: 10616-10623.

National Research Council (NRC). 2012. Nutrient Requirements of swine. 11 th ed. National Academy Press, Washington, DC.

Penz Jr., A.N.; Bruno, D. y Silva, G. 2009. Interação nutrição-reprodução em suínos. Acta Sci Vet, 3: 183-194.

Pupa, J.M.R. y Barroca, C.C. 2013. Efeitos da Nutrição das reprodutoras sobre a progênie. In: $28^{a}$ Reunião Anual do CBNA: Congresso sobre Nutrição de Animais Jovens - Aves e Suínos. Campinas.

Quesnel H.; Pasquier, A.; Mounier, A.M.; Louveau, I. y Prunier, A. 1998. Influence of feed restriction in primiparous lactating sows on body condition and metabolic parameters. Reprod Nutr Dev, 38: 261-274.

Quesnel, H.; Meunier-Salaün, M.C.; Hamard, A.; Guillemet, R.; Etienne, M.; Farmer, C.; Dourmad, J.Y. and Père, M.C. 2008. Dietary fiber for pregnant sows: influence on sow physiology and performance during lactation. J Anim Sci, 87: 532-43.

Quesnel, H. 2009. Nutritional and lactational effects on follicular development in the pig. Soc Reprod Fertil, 66: 121-134.

Quiniou, N.; Mourot, J.; Richard, S.; Etienne, M.; Coudray, L. 2005. Influence de la nature de l'énergie allouée à la truie pendant la gestation et la lactation sur ses performances de lactation et celles de sa portée et sur la composition corporelle des porcs au sevrage et à l'abattage. J Rech. Porcine, 37: 203-210.

Quiniou, N.; Mourot, J.; Étienne, M.; Y Richard, S. 2006. Quel estl'impact d'un apport d'énergie sous forme de lipids pendant la gestation et/ ou la lactation sur les performances des truies allaitantes et celles des porcelets jusqu'à l'abattage? J Rech. Porcine, 38: 177-184.

Rostagno, H. S.; Albino, L. F. T.; Donzele, J. L.; Gomes, P. C.; Oliveira, R. F.; Lopes, D. C.; Ferreira, A. S.; Barreto, S. L. T.; y Euclides, R. F. 2011. Tabelas brasileiras para aves e suínos: composição de alimentos e exigências nutricionais de aves e suínos. $3^{a}$ edição. Viçosa. MG. 252 pp. Silva, B.A.N.; Alcici, P.F.; Araujo, G.G.A.; Fernades, N.S.F,. Rosa, J.F.S.; Florentino, M.S.; Tolentino.; Taveira, V.M. y Pinto, M.F.A. 2014. Recuperação corporal em fêmeas suínas: qual o impacto da perda na lactação sobre a estratégia nutricional a ser adotada na gestação. In: XII Fórum Internacional de Suinocultura, 2014, Foz do lguaçu. Anais... Concórdia, SC : Embrapa Suínos e Aves, p. 58-68.

Sauber, T.E.; Stahly, T.S.; Ewan, R.C. y Williams, R.C. 1994. Interactive effects of sow genotype and dietary amino acid intake on lactational performance of sows nursing large litters. J Anim Sci, 72: 66

Souza, F.A.; Canisso, I.F.; Borges,A.M.; Vale Filho, V.R.; Lima, A.L. y Silva, E.C. 2009. Restrição alimentar e os mecanismos endócrinos associados ao desenvolvimento folicular ovariano em vacas. Rev Bras Reprod Anim, 33: 61-65.

Tokack M.D.; Pettigrew, J.E.; Dial, G.D.; Wheaton, J.E.; Crooker, B.A. y Johnston, L.J. 1992. Characterization of luteinizing hormone secretion in the primiparous lactating sow: relationship to blood metabolites and return-to-estrus interval. J Anim Sci, 70: 2195-2201.

Thingnes, S.L.; Gaustad, A.H.; Kjos, P.N.; Hetland, H. y Framstad, T. 2013. Pea starch meal as a substitute for cereal grain in diets for lactating sows: The effect on sow and litter performance. Livest Sci, 157: 210-217.

Town. S.C.; Putman, C.T.; Turchinsky, N.J.; Dixon, W.T. y Foxcroft, G.R. 2004. Number of conceptuses in utero affects porcine fetal muscle development. Reproduction, 128: 443-454.

Van Den Brand, H.; Dieleman, S.J.; Soede, N.M. y Kemp, B. 2000. Dietary energy source at two feeding levels during lactation of primiparous sows: I. Effects on glucose, insulin, and luteinizing hormone and on 
follicle development, weaning-to-estrus interval, and ovulation rate. J Anim Sci, 78: 396-404.

Waeber, G.; Calandra, T.; Roduit, R.; Haefliger, J.A.; Bonny, C.; Thompson, N.; Thorens, B.; Temler, E.; Meinhardt, A.; Bacher, M.; Metz, C.N.; Nicod, P. y Bucala, R. 1997. Insulin secretion is regulated by the glucose-dependent production of islet $\mathrm{b}$ cell macrophage migration inhibitory factor. Proc Natl Acad Sci USA, 94: 4782-4787.

Webb, R.; Garnsworthy, P.C.; Campbell, B.K. y Hunter, M.G. 2007. Intraovarian regulation of follicular development and oocyte competence in farm animals. Theriogenology, 1: 22-29.

Weldon, W.C.; Lewis, A.J.; Louis, G.F.; Kovar, J.L. y Miller, P.S. 1994. Postpartum hypophagia in primiparous sows:ll. Effects of feeding level during gestation and exogenous insulin on lactation feed intake, glucose tolerance, and epinephrine-stimulated release of nonesterified fatty acids and glucose. J Anim Sci, 72: 395-403.

Wientjes, J.G.M.; Soede, N.M; Laurenssen, B.F.A.; Koopmanschap, R.E.; Van Den Brand, H. y Kemp, B. 2013. Insulin-stimulating diets during the weaning-to-estrus interval do not improve fetal and placental de- velopment and uniformity in high-prolific multiparous sows. Animal, 7: 1307-1316.

Yaseen, M.A.; Wrenzycki, C.; Herrmann, D.; Carnwath, J.W. y Niemann, H. 2001. Changes in the relative abundance of mRNA transcripts for insulin-like growth factor (IGF-I and IGF-II) ligands and their receptors (IGF-IR/IGF-IIR) in preimplantation bovine embryos derived from different in vitro systems. Reproduction, 122: 601-610.

Yin, F.; Wang, J.; Ruan, Z.; Huang, R.; Wang, Y.; Shao, H.; Huang, J.; $M u, Y$.; $y$ Wen, $Q .2012$. The in vitro digestion rate and in vivo digestibility of raw starches from selected cereals and tubers. J Food Agri Environ, 10: 577-581.

Zangeronimo, M. G.; Oberlender, G. y Murgas, L.D.S. 2013. Efeito da nutrição na reprodução em marrãs. Rev Cient Eletrônica Med Vet, 20: 1-20.

Zhao, Y.; Flowers, W.L.; Saraiva, A.; Yeum, K.J. y Kim, W.S. 2013. Effect of social ranks and gestation housing systems on oxidative stress status, reproductive performance, and immune status of sows. J Anim Sci, 91: 5848-5858. 